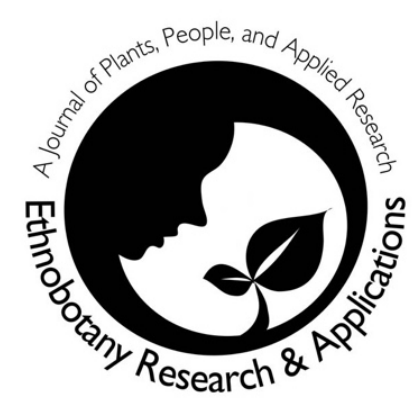

\title{
Talabartería y tinturas de la Sierra de Ancasti, Catamarca, Argentina Saddlery and dyeing in the Sierra de Ancasti, Catamarca, Argentina
}

\author{
Gustavo J. Martínez
}

\section{Research}

\section{Resumen}

Antecedentes: Las plantas curtientes y tintóreas han concitado el interés de diferentes investigaciones en la temática vinculada a la talabartería y la tejeduría tradicional, dos formas tradicionales de labor en creciente desuso. El objetivo de este trabajo es documentar la etnobotánica relacionada con especies tintóreas y curtientes de la sierra de Ancasti, Catamarca, Argentina.

Métodos: Se seleccionaron 46 unidades domésticas a las que se aplicaron encuestas etnobotánicas generales; de manera particular fueron escogidos intencionalmente según la experticia cuatro informantes claves que, de acuerdo a su oficio aportaron información específica y en profundidad sobre talabartería, tejeduría y tinturas. Se trabajó con las fases del trabajo etnobotánico: trabajo de campo y de laboratorio, recolectando e identificando especies vegetales curtientes tintóreas y de interés en cultura material.

Resultados: Un total de 34 especies pertenecientes a 22 familias botánicas comprenden el ámbito de la cultura material, curtiente y tintórea, siendo la familia Fabaceae la más representada (7 spp.). En general nuestras observaciones dieron cuenta del empleo de bastidores y marcos. Menos frecuente es el empleo del telar criollo. La preparación de la tinción es un proceso que involucra a toda la familia siendo una labor considerada localmente como femenina.

Conclusiones: La práctica del teñido natural está todavía vigente en Ancasti y constituye una actividad promisoria. La actividad tintórea y de talabartería se encuentra en el conocimiento y manos de pocos pobladores por lo que sería deseable se desarrollen instancias participativas en las que se puedan compartir y hacer circular estos saberes locales.

Palabras claves: plantas curitentes, tejeduría, tintes, Noroeste Argentino

\section{Correspondence}

Gustavo J. Martínez

IDACOR (Conicet) - Museo de Antropología (UNC). Hipólito Yrigoyen 174. 5000 Córdoba, Argentina

Corresponding Author: gustmart@yahoo.com

Ethnobotany Research \& Applications

20:20 (2020)

\section{Extended Abstract}

Background: Tanning and dyeing plants have attracted the interest of different researches on the subject related to saddlery and traditional weaving, two traditional forms of labor in increasing disuse. The objective of this work is to document the ethnobotany related to dyeing and tanning species from the Sierra de Ancasti, Catamarca, Argentina. The specifics aims are to characterize relevant aspects of Creole saddlery, to characterize relevant species in the material culture associated with equestrian implements and to make a list of dyeing and tanning species used by the "criollo" peasant population

Methods: The Ancasti mountain range is located in the east of the province of Catamarca and is considered an area of great biodiversity where the following ecoregions converge: Yungas (Selva de 
Transición, Selva Montana) [Yungas]; Monte de Sierras y Bolsones [Argentine Northwest Monte]; and Chaco Seco (Chaco Serrano) [Dry Chaco]. The community defines itself as "Criollos-serranos" and its subsistence economy is associated with traditional livestock production systems (cattle, goats, and sheep) and small-scale agriculture, mainly maíz (corn) (Zea mays L.) and zapallo (squash) (Cucurbita maxima Duch.). Forty-six household units were selected to which general ethnobotanical surveys were applied; in particular four key informants were intentionally chosen according to their expertise and their profession, provided specific and in-depth information on saddlery, weaving and dyeing. We worked with the phases of ethnobotanical work: field and laboratory work, collecting and identifying dye tanning plant species of interest in material culture.

Results: A total of 34 species belonging to 22 botanical families comprise the material, tanning and dyeing culture, with the Fabaceae family being the most represented (7 spp.). In general, our observations showed the use of racks (for overcoat) and frames (for making rugs) and for the manufacture of other small pieces such as belts, peleros and caronillas. Less frequent is the use of the Creole loom. Staining preparation is a process that involves the entire family despite being a rather feminine task.

Conclusions: The practice of natural dyeing is still in force at Ancasti and is a promising activity. However, dyeing and saddlery activities are in the knowledge and hands of few residents, so it would be desirable to develop participatory instances in which this local knowledge can be shared and circulated.

Keywords: tanning plants, weaving, dyes, Argentine Northwest

\section{Antecedentes}

La búsqueda de riquezas mineras sobre todo metales preciosos como el oro y la plata constituyó el gran objetivo de la corona española y de los conquistadores que arribaron a partir del descubrimiento de América. Una vez agotada la actividad minera los conquistadores comenzaron a realizar pruebas con la agricultura y la ganadería. En la época indígena, la ganadería estaba compuesta por los camélidos americanos donde sobresalía por su utilidad como animal de carga y proveedor de lana y carne de llama. Los españoles introdujeron después los animales de Castilla: oveja (Ovis orientalis), vacas (Bos taurus), caballos (Equus ferus) y asnos (Equus africanus) (www.atlas.catamarca.gob.ar). Al igual que en otras regiones de Argentina, la introducción del ganado desde la época de la conquista tuvo un papel muy destacado en la consolidación de los primeros asentamientos humanos formados por los españoles y en su desarrollo económico y cultural a través de la provisión de alimento (carne y leche), abrigo (cuero y lana) y trabajo (transporte) (Martínez et al. 2000). En este sentido, el área de sierra en Catamarca se fue convirtiendo en la zona ganadera por excelencia de esta provincia. El impulso de la actividad ganadera se dio con la introducción de vacas, ovejas y yeguarizos que se multiplicaban con gran facilidad en las estancias de los españoles. Estas actividades permitieron la prosperidad, de manera que se resolvió el problema de la subsistencia, y además permitió el intercambio comercial entre las regiones, fundamentalmente con el Potosí. (www.atlas.catamarca.gob.ar). El aprovechamiento del ganado introducido ha dado lugar a diferentes industrias derivadas de estos productos tales como las de la carne, del cuero, de los tejidos, entre otras a las que este trabajo hará referencia para contextualizar y dar cuenta del uso de plantas tintóreas, curtientes y en cultura material.

La talabartería constituye un oficio que se ocupa de la confección de arreos y guarniciones en general para animales de silla y tiro (cabestros, cinchas, riendas, bozales, colleras, monturas, etc.) como también de complementar la terminación en los vehículos en todas aquellas partes que requieren la utilización del cuero y de la suela (Musarra 2006).

Un capítulo particular dentro de la talabartería merecen las materias curtientes, esto es aquellas sustancias que tienen la propiedad de que sus soluciones, al ser absorbidas por las pieles de los animales, las transforman en cueros; para el caso, las plantas ricas en taninos suelen ser un buen material curtiente (Marzocca 1959, 1993).

Asimismo un tema de interés generalizado en etnobotánica y botánica económica es el de las plantas tintóreas. Si bien se da un uso generalizado de fibras y colorantes sintéticos del tipo anilinas, existe un interés creciente en los últimos años en un teñido basado en el empleo de productos naturales especialmente vegetales puesto que aventaja a los procedimientos modernos en aspectos como resistencia a los colores, posibilidades de abastecimiento y bajo impacto ambiental (Trillo et al. 2007; Trillo \& Demaio 2007).

La temática de las plantas tintóreas y colorantes ha sido abordada en diferentes grupos culturales y étnicos en Argentina. Así Suárez \& Arenas (2012) dan cuenta de los tintes vegetales y fúngicos entre los indígenas wichí del Chaco Semiárido para el teñido de las fibras de chaguar. Por su parte Arenas 
(2004) y Keller (2010) refieren, respectivamente, una serie de productos colorantes y tintóreos entre los indígenas del Gran Chaco y de Misiones tanto de uso ornamental y corporal (en tatuajes) como textil. De las regiones del Alto Bermejo y Yungas traen referencias acerca de este tópico los trabajos de Lambaré et al. (2011) y Hilgert (2007). Para el centro de Argentina la temática de las plantas tintóreas ha sido tratada por Paván et al. (2017) y por Trillo et al. (2007) y para la provincia de Córdoba y por Palacio et al. (2007) y Palacio (2018) para Santiago del Estero. Por su parte para toda Argentina el tratamiento de esta temática se dio en forma general en publicaciones que dan cuenta de listados de especies y técnicas de coloración, hilado y tejido (Joseau et al. 2018, Marzocca 1959, 1993, Stramigioli 1991, Trillo \& Demaio 2007). En lo que concierne a la provincia de Catamarca, si bien existe una serie de contribuciones sobre el uso de la seda nativa de Lepidópteros (Jurado \& Zapata 2002, Zapata \& Jurado Cazaux 2018 a,b) no encontramos antecedentes de publicaciones que aborden el uso de plantas tintóreas ni curtientes, por lo que este trabajo pretende cubrir este espacio académico.

En este contexto, los objetivos de este trabajo son: Caracterizar aspectos relevantes de la talabartería criolla; identificar las especies más importantes en la cultura material asociada con implementos hípicos y enlistar las especies tintóreas y curtientes usadas por la población criolla campesina de la región.

\section{Materiales y Métodos}

\section{Área y población de estudio}

El área de estudio se encuentra en el Departamento de Ancasti, el que se ubica al este de la provincia de Catamarca (Figura 1), abarcando asimismo el cordón montañoso de Ancasti, el que supera los $1200 \mathrm{~m}$ de altitud y se considera un área de gran biodiversidad por la confluencia de diferentes ecorregiones, las Yungas (Selva de Transición, Selva Montana); el Monte de Sierras y Bolsones; y el Chaco Seco (Chaco Serrano) (Maldonado 2011, Morláns 1995, Palmeri et al. 2008). En cuanto a la estructura vegetal la zona se caracteriza por combinar elementos arbóreos típicos del Chaco Semiárido, como Celtis spp., Geoffroea decorticans (Gillies ex Hook. \& Arn) Burkart., Jodina rhombifolia (Hook. \& Arn.) Reissek y Schinopsis lorentzii (Griseb.) Engl, con elementos propios del Chaco Serrano, como Parapiptadenia excelsa (Griseb.) Burkart, Prosopis nigra (Griseb.) Hieron. y Zanthoxylum coco Gillies ex Hook. f. \& Arn., y especies más relacionadas a la Selva de Transición, como Anadenanthera colubrina (Vell.) Brenan, Erythroxylum argentinum O.E. Schulz y Xylosma pubescens Griseb.

Según los datos más recientes datos del Instituto Nacional de Estadísticas y Censos (INDEC 2010), el departamento de Ancasti está conformado por una población de 2900 habitantes, que conforman un total de 786 hogares o unidades familiares. La comunidad se autodefine como "criollos-serranos" y su economía de subsistencia está asociada a sistemas de producción de ganadería tradicional (bovina, caprina y ovina) y agricultura a pequeña escala principalmente de maíz (Zea mays L.) y zapallo (Cucurbita maxima Duch.).

Jiménez-Escobar (2019 a,b) citando a Bazán (2006) refiere cómo, desde la segunda mitad del siglo XVIII (1765) y durante todo el siglo XIX la ganadería y la curtiembre de suelas fueron las producciones socioeconómicas más representativas de Ancasti. El autor señala a su vez que "para esa época, la cría de ganado (yeguas, mulas, cabras y ovejas) se vendían para el consumo en las provincias de Córdoba, San Juan e incluso llevadas al país limítrofe de Chile; por otro lado, la curtiembre de suelas se comercializaba con las provincias de Buenos Aires, Córdoba, Cuyo y Tucumán; además el aprovechamiento del cuero era también la base de una artesanía local muy estimada en el litoral: La confección de monturas, riendas y cinchas (para el trasporte en lomo de mula o caballo)".

Como vemos en la Sierra de Ancasti era muy importante la artesanía en cuero. Además del curtido de cueros y suelas también se fabricaban petacas, roperos, monturas, lazos y riendas. La Sierra se fue convirtiendo en la zona ganadera por excelencia. Se llevaban a Salta las mulas a una feria anual para su venta en los meses de marzo-abril; las vacas se vendían en Córdoba y Chile. Ese tipo de comercio subsistió en el siglo pasado, interrumpiéndose solamente durante la guerra de la emancipación. (www.atlas.catamarca.gob.ar). Para inicios del siglo XX la carne de la Pampa húmeda superaba ampliamente en calidad a la de los rodeos locales, por lo que no pudo competir con ésta, por lo que se vio reducido drásticamente el mercado (JiménezEscobar 2019 a, b). No contamos con datos precisos sobre lo que ocurrió con la industria talabartera y textil, pero es muy probable que haya quedado confinada al uso local, algo que todavía en la actualidad se mantiene en muy pocos casos y especialistas, como veremos en los resultados. 


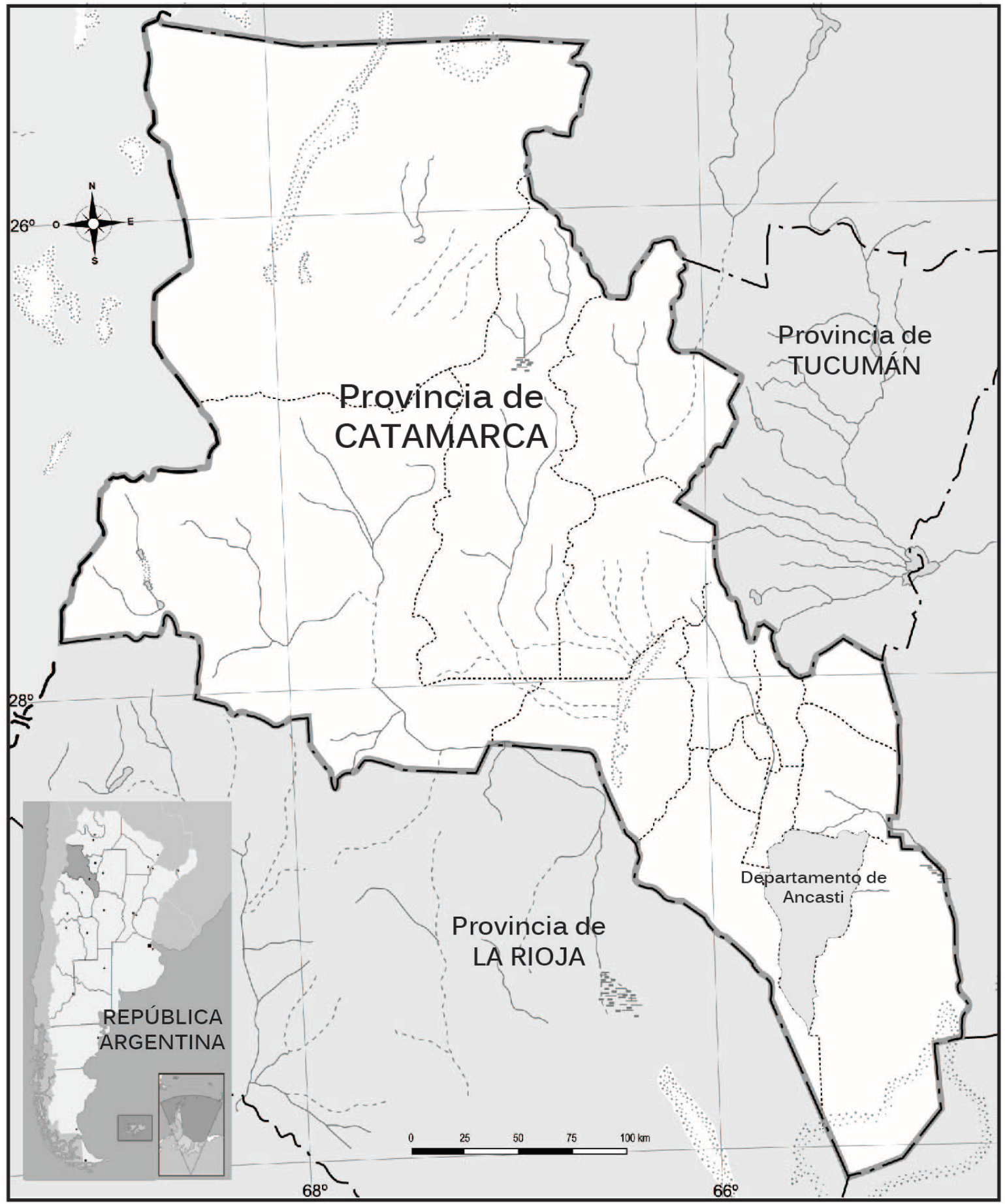

Figura 1. Localización del área de estudio, departamento de Ancasti, provincia de Catamarca, Argentina. Figure 1. Location of the study area, Ancasti department, Catamarca province, Argentina.

Debemos señalar finalmente en cuanto a los procesos poblacionales de estas comunidades, en la actualidad se observa una reciente migración de pequeños productores catamarqueños hacia centros urbanos debido, entre otras razones, a los cambios en la producción y en el uso de la tierra (Jiménez-Escobar 2019 b, Jiménez-Escobar \& Martínez 2019).

\section{Viajes de trabajo de recolección de datos etnobotánicos}

Se utilizaron técnicas de uso habitual en etnobiología (Albuquerque et al. 2010, Anderson et al. 2011, Martin 2001). Previo a las entrevistas con los pobladores, se informó acerca del proyecto de investigación y sus objetivos a los representantes municipales y se contó con la aprobación de la Secretaría de Ambiente y Desarrollo de la provincia de Catamarca. Así mismo se informó a los miembros 
de la comunidad e individualmente en cada unidad doméstica en la que se solicitó el consentimiento verbal de acuerdo con el código de ética de la International Society of Ethnobiology (ISE 2006). En total 46 unidades domésticas participaron de la investigación etnobotánica general (87 habitantes entre los 23 y los 80 años) la que incluía preguntas generales acerca de la temática en el marco de un trabajo amplio sobre la etnobotánica campesina; de manera particular fueron seleccionadas intencionalmente según la experticia cuatro informantes claves que, de acuerdo a su oficio aportaron información específica y en profundidad sobre talabartería, tejeduría y tintorería.

La información que atañe a la temática se obtuvo en diferentes instancias del proceso de investigación, a saber:

a) Registros y documentación de especies, nombres vernáculos y usos en general, en el marco de caminatas etnobotánicas con informantes calificados.

b) Entrevistas abiertas y en profundidad

c) Encuestas semiestructuradas específicas

d) Observación participante.

El material de las entrevistas se registró en grabaciones digitales y en cuadernos de campo. Las plantas fueron recolectadas principalmente en estado reproductivo para su posterior identificación. La colección fue procesada según los estándares establecidos (Alexiades 1996). Los ejemplares fueron determinados por el autor y posteriormente depositados en el Herbario del Museo Botánico (CORD), del Instituto Multidisciplinario de Biología Vegetal (IMBIV), Universidad Nacional de Córdoba, Argentina, bajo la serie de numeración G.J. Martínez y N.D. Jiménez-Escobar. La información etnobotánica se sistematizó en una base de datos Excel.

\section{Análisis de datos}

Los datos de los cuadernos de campo permitieron generar categorías de análisis de la información local clasificados en los siguientes usos: tecnologías locales (se refieren a resinas, jabones e implementos para el curtido del cuero como bateas); cultura material (asociada a implementos hípicos como madera para monturas); curtientes (para el tratamiento del cuero) y tintóreos (para el tratamiento de la lana).

\section{Resultados y Discusión \\ Aspectos generales}

Un total de 34 especies pertenecientes a 22 familias botánicas comprenden el ámbito de la cultura material, curtiente y tintórea, siendo la familia Fabaceae la más representada (7 $\begin{array}{lll}7 & \text { spp.). }\end{array}$
Consideramos que esto es así porque esta familia contiene especies con más aplicaciones etnobotánicas y más diversificados en los usos que otras familias; además presentan riqueza de alcaloides y sus frutos al ser legumbres se destacan, duran mucho tiempo en la planta (lo que los hace vistosos y perceptibles) y no se pudren con facilidad, lo que permite verlas en estado seco $y$ almacenarlas. Debemos señalar, asimismo, que Fabaceae, junto con Asteraceae y Poaceae constituyen las familias de mayor riqueza en los ecosistemas de Argentina (Cantero et al. 2019).

De estas 34 especies, 26 son nativas y las 8 restantes exóticas, dando cuenta de la preservación de un conocimiento tradicional en la temática y de la generación de un conocimiento híbrido en estos campos del conocimiento etnobotánico, especialmente en lo que concierne a las plantas tintóreas. Es importante señalar que la cría de ganado para cuero ha sido una actividad muy importante desde tiempos de la colonia y esto ha generado un conocimiento especializado en la temática fruto especialmente de la experiencia con plantas nativas del lugar.

En relación con las partes utilizadas predominan el uso de las cortezas (9 usos; $27 \%$ ), seguido de las maderas $(24 \%)$, hojas (18\%), raíces (15\%), parte aérea $(12 \%)$ y resinas $(3 \%)$.

\section{Cueros, curtientes y cultura material}

Uno de los aspectos fuertemente vinculados al mantenimiento del curtido tradicional son las tradiciones ecuestres. Los pobladores de Ancasti refieren su participación en la Fiesta de Jinetes, caballos y aperos organizados por la localidad de Las Juntas, Departamento Ambato, Provincia de Catamarca. El mismo consiste en desfile de agrupaciones gauchas, artesanías tradicionales y comidas típicas, así como desfiles de razas y destrezas equinas. Otra festividad relacionada con la temática es la fiesta del poncho que se organiza todos los inviernos en la capital de Catamarca, allí se presentan todo tipo de artesanías incluso las de tejeduría.

En relación con la cultura material los informantes dieron cuenta de los componentes del apero. De acuerdo con su colocación, de inferior a superior encontramos en primer lugar el pelero, los sobrepelos, la carona de suela, la montura con estribos, la cincha, los pellones, el sobrepuesto y otra cincha más o encimera de cuero. Otros materiales que se ubican en la cabeza del animal son las cabezadas y riendas que son de suela 0 cuero trenzado, y el freno, que es de hierro. En el mismo contexto, entre los útiles más frecuentemente utilizados por el talabartero encontramos la lezna, el 
punzón, la escofina, las pinzas de costura, el sacabocados, la trincheta y la piedra de afilar.

\section{El curtido y sus aplicaciones}

Con respecto a la elaboración y el curtido de cuero, los pobladores relatan los siguientes pasos en la elaboración de lazos y riendas.

1. Lazos: Cabestros de cuero crudo de 6-9 brazadas de longitud (9 $\mathrm{m}$ aproximadamente). El proceso consiste en: Estaquear el cuero para lograr que se seque; seguidamente se humedecen los correones o tiras que se sacan, ya sea mojándolas en agua y estirándolos entre estacas, previo pelado con cuchillo y en otros casos con cal (en este último caso no es muy útil porque el cuero queda demasiado duro y se resquebraja). Posteriormente y una vez seco, nuevamente se lo empareja o arregla para que queden con un mismo tamaño, utilizándose para ello un cuchillo. Se lo vuelve a mojar en agua, se lo envuelve en una lona de tela, se forma una especie de madeja para trenzar, se pone en una argolla y según sea de 4,6,8,5 hilos trenzados se pone un botón que es donde pasa la presilla. Seguidamente se procede al trenzado. Se cura con plantas (corteza de sombra de toro -Jodina rhombifolia-, malva Sphaeralcea bonariensis- o tuna -Opuntia ficusindica) para aflojar y sobar el cuero. Finalmente se estira para que se seque.

2. Suela para aperos: Apenas se carnea el animal se lo pone en agua y cal (8 días suficientes para ablandarlo). Luego se lo pela. Se lava en agua y raspa con cuchillo de ambos lados para que salgan los restos de cal y pelo. Posteriormente, se trae corteza de cebil (Anadenanthera colubrina var. cebil) molido en un mortero y se lo coloca en una pileta con agua donde se ubicará el cuero; hay que apretarlo lo suficiente para que salga el aire, y en unos 10 días aproximadamente se da vuelta el cuero para que el curtido resulte parejo, ya que donde queda aire se impide el ingreso de la solución curtiente y un adecuado curtido. Para obtener una buena suela, según el recipiente empleado y el grosor del cuero se requiere un macerado de 24 a 30 días. Para evaluar el avance del curtido se corta una lonjita de cuero y se observa la coloración interna del mismo. Si aún está de color blanco, significa que falta macerado y que aún no absorbió bien el tinte propio de la solución curtiente. Una vez completada la preparación del cuero para suelas, se lo saca del recipiente, se lava y se cura con grasa de gallina y/o aceite especial y se lo seca. Con la suela se preparan distintos elementos asociados a actividades ecuestres: la caronilla, el sobrepuesto, la carona, las riendas y el pelero.

3. Aperos: Por lo general la gente del lugar emplea el apero con elevaciones o puntas en las caronas. Es común que en su elaboración se emplean maderas livianas -que no dañen al animal-, como las de lecherón (Sapium haematospermum), pacará (Enterolobium contortisiliquum), sauce (Salix humboldtiana), brea (Parkinsonia praecox), cardón moro (Stetsonia coryne) (Tabla 1; Figura 2). El armado de un apero insume entre 20 y 30 días y su costo varía según el destino; en el año 2015, un apero de campo completo oscilaba los 20 mil pesos (u\$200); mientras que un apero de desfile los 40 a 50 mil pesos (entre US\$ 400 a 500). La Figura 2 muestra alguno de los implementos hípicos y una montura de tipo salteña.

Tabla 1. Especies y usos curtientes, tintóreos y en cultura material vinculados al manejo del ganado.

Table 1. Species and uses in tanning, dyeing and material culture related to livestock management

\begin{tabular}{|c|c|c|c|}
\hline Especie (Familia) Voucher & $\begin{array}{l}\text { Nombre } \\
\text { vernáculo }\end{array}$ & Uso & Detalle de usos \\
\hline $\begin{array}{l}\text { Anadenanthera colubrina } \\
\text { (Vell.) Brenan var. cebil } \\
\text { (Griseb.) Altschul } \\
\text { (Fabaceae) } \\
\text { GJM 925, } 1191\end{array}$ & Cebil & Curtiente & $\begin{array}{l}\text { La corteza se utiliza para el curtido } \\
\text { de cueros. }\end{array}$ \\
\hline $\begin{array}{l}\text { Baccharis flabellata Hook. \& } \\
\text { Arn. } \\
\text { (Asteraceae) } \\
\text { NDJ } 2245\end{array}$ & Clavillo & Tintóreo & Las hojas dan un tinte color amarillo. \\
\hline $\begin{array}{l}\text { Berberis ruscifolia Lam. } \\
\text { (Berberidaceae) } \\
\text { GJM } 1288\end{array}$ & Chilca & Tintóreo & Las hojas dan un tinte color amarillo. \\
\hline $\begin{array}{l}\text { Beta vulgaris } L \text {. } \\
\text { Amaranthaceae }\end{array}$ & Remolacha & Tintóreo & $\begin{array}{l}\text { La raíz da un tinte color rojizo } \\
\text { morado. }\end{array}$ \\
\hline $\begin{array}{l}\text { Bidens sp. } \\
\text { (Asteraceae) }\end{array}$ & Amor seco & Tintóreo & $\begin{array}{l}\text { La decocción de la parte aérea da un } \\
\text { tinte color amarillento. }\end{array}$ \\
\hline
\end{tabular}




\begin{tabular}{|c|c|c|c|}
\hline $\begin{array}{l}\text { Celtis tala Gillies ex Planch. } \\
\text { (Cannabaceae) } \\
\text { GJM } 905\end{array}$ & Tala & Cultura material & Su madera es apta para chicotes. \\
\hline $\begin{array}{l}\text { Condalia spp. } \\
\text { (Rhamnaceae) } \\
\text { GJM 940, } 1290\end{array}$ & Piquillín & Tintóreo & La raíz da un tinte color rojo. \\
\hline $\begin{array}{l}\text { Daucus carota } L . \\
\text { Apiaceae }\end{array}$ & Zanahoria & Tintóreo & $\begin{array}{l}\text { La raíz da un tinte color amarillo } \\
\text { anaranjado. }\end{array}$ \\
\hline \multirow[t]{2}{*}{$\begin{array}{l}\text { Enterolobium } \\
\text { contortisiliquum ( } \\
\text { Morong } \\
\text { (Fabaceae) } \\
\text { GJM } 1240\end{array}$} & Pacará & Cultura material & $\begin{array}{l}\text { La madera se emplea para } \\
\text { monturas. }\end{array}$ \\
\hline & & Tintóreo & $\begin{array}{l}\text { No se pudo documentar el color que } \\
\text { da su tinte. }\end{array}$ \\
\hline $\begin{array}{l}\text { Geoffroea decorticans } \\
\text { (Gillies ex Hook. \& Arn.) } \\
\text { Burkart } \\
\text { (Fabaceae) } \\
\text { NDJ } 2219\end{array}$ & Chañar & Cultura material & $\begin{array}{l}\text { La madera se emplea para } \\
\text { monturas. Se emplean los codos de } \\
\text { estos árboles para las porciones } \\
\text { superiores donde la montura } \\
\text { requiere dureza. }\end{array}$ \\
\hline $\begin{array}{l}\text { Heimia salicifolica (Kunth) } \\
\text { Link (Lythraceae) } \\
\text { GJM } 855\end{array}$ & $\begin{array}{l}\text { Arupaco, } \\
\text { quiebrarado }\end{array}$ & Tintóreo & $\begin{array}{l}\text { Se emplea la totalidad de la planta, } \\
\text { la que da una tonalidad gris. Los } \\
\text { nativos aprendieron este uso de la } \\
\text { población de artesanos neorrurales. }\end{array}$ \\
\hline $\begin{array}{l}\text { Ilex paraguariensis } \\
\text { A.St.-Hil. } \\
\text { Aquifoliaceae }\end{array}$ & Yerba mate & Tintóreo & $\begin{array}{l}\text { El producto comercial (hojas } \\
\text { molidas) da tintes amarillos } \\
\text { verdosos. }\end{array}$ \\
\hline $\begin{array}{l}\text { Jatropha macrocarpa } \\
\text { Griseb. } \\
\text { (Euphorbiaceae) } \\
\text { GJM } 1237\end{array}$ & $\begin{array}{l}\text { Piñón, higuera } \\
\text { del zorro }\end{array}$ & Tintóreo & $\begin{array}{l}\text { La corteza se emplea para teñir y la } \\
\text { savia marca los cueros dándoles un } \\
\text { tinte grisáceo. }\end{array}$ \\
\hline $\begin{array}{l}\text { Jodinia rhombifolia (Hook. \& } \\
\text { Arn.) Reissek } \\
\text { (Cervantesiaceae) } \\
\text { GJM } 914\end{array}$ & Sombra de toro & Curtiente & $\begin{array}{l}\text { La corteza en leche sirve para curar } \\
\text { y lavar los lazos. }\end{array}$ \\
\hline $\begin{array}{l}\text { Juglans australis Griseb. } \\
\text { (Juglantaceae) } \\
\text { GJM } 1046\end{array}$ & $\begin{array}{l}\text { Nogal, nogal } \\
\text { cimarrón }\end{array}$ & Tintóreo & $\begin{array}{l}\text { La corteza, las hojas y las ramas dan } \\
\text { tintes marrones oscuros. }\end{array}$ \\
\hline $\begin{array}{l}\text { Juglans regia L. } \\
\text { (Juglantaceae) }\end{array}$ & Nogal & Tintóreo & $\begin{array}{l}\text { La corteza da un tinte en los tonos } \\
\text { marrones oscuros. }\end{array}$ \\
\hline \multirow[t]{2}{*}{$\begin{array}{l}\text { Myrcianthes cisplatensis } \\
\text { (Cambess.) O. Berg } \\
\text { (Myrthaceae) } \\
\text { GJM 895, } 1044\end{array}$} & Huil, mato huil & Tintóreo & Raíz y corteza dan un tinte marrón. \\
\hline & & Cultura material & $\begin{array}{l}\text { La madera se emplea para hacer } \\
\text { sujetador de telar. }\end{array}$ \\
\hline $\begin{array}{l}\text { Parasenegalia visco } \\
\text { (Lorentz ex Griseb.) Seigler } \\
\text { \& Ebinger } \\
\text { (Fabaceae) } \\
\text { GJM } 1053\end{array}$ & Viscote & Tintóreo & $\begin{array}{l}\text { Su corteza da un tinte marrón } \\
\text { oscuro. }\end{array}$ \\
\hline $\begin{array}{l}\text { Parkinsonia praecox (Ruiz \& } \\
\text { Pav. ex Hook.) Hawkins } \\
\text { (Fabaceae) }\end{array}$ & Brea & $\begin{array}{l}\text { Tecnologías } \\
\text { locales }\end{array}$ & $\begin{array}{l}\text { Se queman troncos y/o ramas y se } \\
\text { extrae cenizas con las que se } \\
\text { elaboran jabones caseros. }\end{array}$ \\
\hline GJM 1221 & & $\begin{array}{l}\text { Tecnologías } \\
\text { locales }\end{array}$ & $\begin{array}{l}\text { Se extrae una resina gomosa } \\
\text { mediante tajos en la corteza, que }\end{array}$ \\
\hline
\end{tabular}




\begin{tabular}{|c|c|c|c|}
\hline & & & $\begin{array}{l}\text { sirve de pegamento para papel y } \\
\text { para consumo. }\end{array}$ \\
\hline & & Cultura material & $\begin{array}{l}\text { La madera se emplea para } \\
\text { monturas. Se le atribuyen las } \\
\text { propiedades de ser "buena, liviana y } \\
\text { blanca". }\end{array}$ \\
\hline $\begin{array}{l}\text { Prosopis sericantha Gillies } \\
\text { ex Hook. \& Arn. } \\
\text { (Fabaceae) } \\
\text { GJM 1217, } 1229\end{array}$ & Albardón & Cultura material & $\begin{array}{l}\text { Con la raíz se preparan cabezas de } \\
\text { leznas de talabartería, mangos de } \\
\text { chicotes y cabos de cuchillos. }\end{array}$ \\
\hline \multirow{2}{*}{$\begin{array}{l}\text { Prosopis spp. (Fabaceae) } \\
\text { GJM } 1216 \\
\text { GJM } 949\end{array}$} & Algarrobo & Tintóreo & $\begin{array}{l}\text { La corteza da un tinte color marrón } \\
\text { oscuro. }\end{array}$ \\
\hline & & Cultura material & $\begin{array}{l}\text { La madera se emplea en estribos } \\
\text { para caballos. También para } \\
\text { sujetador, envolvedor, palita y peine } \\
\text { de telares. }\end{array}$ \\
\hline $\begin{array}{l}\text { Punica granatum L. } \\
\text { (Lythraceae) }\end{array}$ & Granada & Tintóreo & $\begin{array}{l}\text { La corteza de sus frutos con hierro } \\
\text { da un tinte negro. }\end{array}$ \\
\hline $\begin{array}{l}\text { Salix humboldtiana Willd. } \\
\text { (Salicaceae) } \\
\text { GJM } 929\end{array}$ & Sauce llorón & Cultura material & $\begin{array}{l}\text { La madera se emplea para } \\
\text { monturas. }\end{array}$ \\
\hline $\begin{array}{l}\text { Sapium haematospermum } \\
\text { Müll. Arg. } \\
\text { (Euphorbiaceae) } \\
\text { GJM } 928\end{array}$ & $\begin{array}{l}\text { Lechico, } \\
\text { lecherón }\end{array}$ & Cultura material & $\begin{array}{l}\text { La madera se utiliza para bastos de } \\
\text { monturas, aunque requiere curarse } \\
\text { de las polillas. }\end{array}$ \\
\hline $\begin{array}{l}\text { Schinopsis lorentzii (Griseb.) } \\
\text { Engl. } \\
\text { (Anacardiaceae) } \\
\text { GJM } 1392\end{array}$ & $\begin{array}{l}\text { Quebracho } \\
\text { colorado }\end{array}$ & Tintóreo & $\begin{array}{l}\text { Distinguen dos variedades: el } \\
\text { "campana" que da un tinte marrón } \\
\text { oscuro, de hojas de mayor tamaño y } \\
\text { fruto más claro; y el "llanisto" que da } \\
\text { un tinte verde claro y es de hojas } \\
\text { más pequeñas. }\end{array}$ \\
\hline $\begin{array}{l}\text { Schinus piliferus I.M. Johnst. } \\
\text { (Anacardiaceae) } \\
\text { GJM } 1252\end{array}$ & $\begin{array}{l}\text { Molle tonto } \\
\text { curtidor, molle } \\
\text { de curtir, molle } \\
\text { sonso }\end{array}$ & $\begin{array}{l}\text { Curtiente y } \\
\text { tratamiento del } \\
\text { cuero }\end{array}$ & $\begin{array}{l}\text { La corteza es curtiente y se emplea } \\
\text { en el tratamiento del cuero (trenzas, } \\
\text { lasos, bastos, rebenques, cinchas, } \\
\text { cabestros, riendas, maniadores). } \\
\text { Afirma el color a la suela. }\end{array}$ \\
\hline $\begin{array}{l}\text { Sphaeralcea bonariensis } \\
\text { (Cav.) Griseb. } \\
\text { (Malvaceae) } \\
\text { GJM } 854\end{array}$ & $\begin{array}{l}\text { Malva, malva } \\
\text { blanca }\end{array}$ & $\begin{array}{l}\text { Curtiente y } \\
\text { tratamiento del } \\
\text { cuero }\end{array}$ & $\begin{array}{l}\text { Se remojan las hojas en leche para } \\
\text { curar y limpiar los lazos. Después de } \\
\text { curtir las suelas se le pasa malva } \\
\text { para curar. La malva se restriega en } \\
\text { la soga, queda verde y se cura el } \\
\text { lazo; es como si se le pusiera grasa, } \\
\text { queda más blando. }\end{array}$ \\
\hline $\begin{array}{l}\text { Stetsonia coryne (Salm- } \\
\text { Dyck) Britton \& Rose } \\
\text { (Cacataceae) } \\
\text { GJM } 1099\end{array}$ & Cardón moro & Cultura material & $\begin{array}{l}\text { La madera se emplea para hacer } \\
\text { monturas. Se considera que es de } \\
\text { un material liviano que no lastima el } \\
\text { animal. }\end{array}$ \\
\hline $\begin{array}{l}\text { Tripodanthus acutifolius } \\
\text { (Ruiz \& Pav.) Tiegh. } \\
\text { (Loranthaceae) } \\
\text { GJM957, } 1264\end{array}$ & Corpo, corpus & Cultura material & $\begin{array}{l}\text { La madera es apta para chicotes y } \\
\text { mangos de herramientas. }\end{array}$ \\
\hline $\begin{array}{l}\text { Urtica urens L. } \\
\text { (Urticaceae) } \\
\text { GJM } 1315\end{array}$ & Ortiga & Tintóreo & $\begin{array}{l}\text { La parte aérea da un tinte color } \\
\text { verde amarillo. }\end{array}$ \\
\hline $\begin{array}{l}\text { Usnea amblyoclada (Müll. } \\
\text { Arg.) Zahlbr. }\end{array}$ & Barba de piedra & Tintóreo & $\begin{array}{l}\text { El talo da un tinte color marroncito } \\
\text { claro. }\end{array}$ \\
\hline
\end{tabular}




\begin{tabular}{|c|c|c|c|}
\hline $\begin{array}{l}\text { (Parmeliaceae) } \\
\text { GJM 1033, 1172) }\end{array}$ & & & \\
\hline \multirow{2}{*}{$\begin{array}{l}\text { Ximenia americana L. } \\
\text { (Ximeniaceae) } \\
\text { GJM } 1030\end{array}$} & \multirow[t]{2}{*}{ Pata, albarillo } & Tintóreo & $\begin{array}{l}\text { La raíz da un tinte color gris } \\
\text { negruzco. }\end{array}$ \\
\hline & & Curtiente & $\begin{array}{l}\text { Se emplea para curtir y teñir } \\
\text { recipientes de madera. }\end{array}$ \\
\hline \multirow[t]{2}{*}{$\begin{array}{l}\text { Yucca cfr.aloifolia L. } \\
\text { (Asparagaceae) }\end{array}$} & \multirow[t]{2}{*}{ Yuca } & Tintóreo & $\begin{array}{l}\text { Las hojas dan un tinte amarillo y la } \\
\text { raíz color marrón. }\end{array}$ \\
\hline & & $\begin{array}{l}\text { Tecnologías } \\
\text { locales }\end{array}$ & $\begin{array}{l}\text { Las hojas se muelen y la espuma se } \\
\text { utilizaba como jabón para limpiar la } \\
\text { ropa. }\end{array}$ \\
\hline \multirow{3}{*}{$\begin{array}{l}\text { Zanthoxylum coco Gillies ex } \\
\text { Hook. f. \& Arn. } \\
\text { (Rutaceae) } \\
\text { GJM 902, } 1266\end{array}$} & \multirow[t]{3}{*}{ Coco } & Tintóreo & La corteza da un tinte color amarillo. \\
\hline & & $\begin{array}{l}\text { Curtiente y } \\
\text { tratamiento del } \\
\text { cuero }\end{array}$ & $\begin{array}{l}\text { La corteza se puede usar para } \\
\text { curtiembre, pero sale de otro color la } \\
\text { suela más amarillo -no roja- y } \\
\text { cuando no se quiere que salga tan } \\
\text { rojo se mezcla con cebil. Se usa } \\
\text { porque afirma el color de la suela. }\end{array}$ \\
\hline & & $\begin{array}{l}\text { Tecnologías } \\
\text { locales }\end{array}$ & $\begin{array}{l}\text { La madera se usa para confeccionar } \\
\text { bateas. }\end{array}$ \\
\hline
\end{tabular}

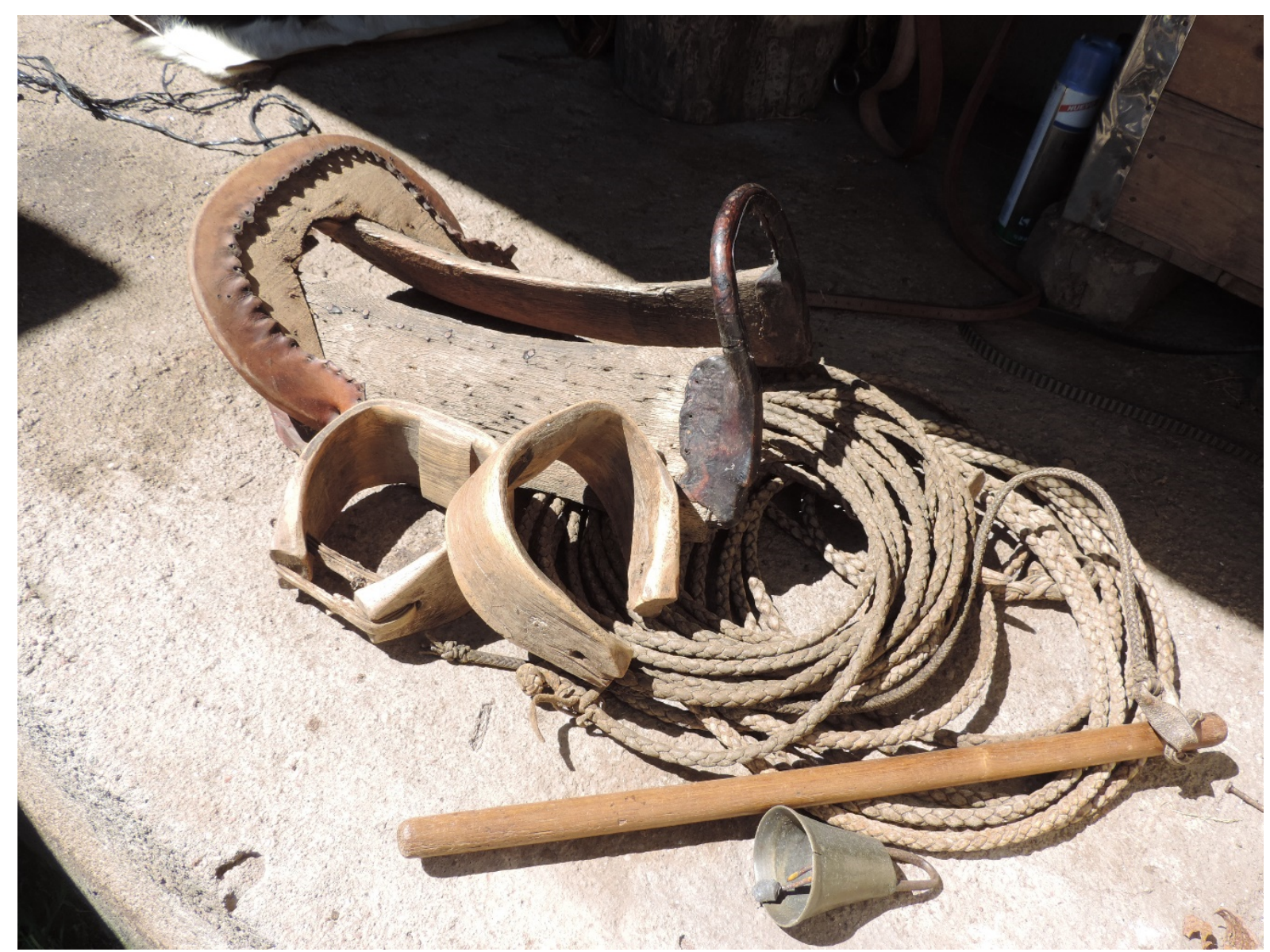

Figura 2. Montura tipo salteña hecha de cardón (Stetsonia coryne, Cactaceae) e implementos hípicos (rebenque, lazos y otros) usados en Ancasti.

Figure 2. Salta type saddle made by "cardón" (Stetsonia coryne, Cactaceae) and riding implements (bridle, ties, and others) used in Ancasti 
En relación con las especies curtientes se encuentran algunas que fueron ya señaladas por Marzocca $(1959,1993)$ y Hilgert (2007) como el coco -Zanthoxylum coco- y el cebil -Anadenanthera colubrina var. cebil- mientras otras constituyen novedades en la literatura como la pata -Ximenia americana- y la malva -Sphaeralcea bonariensis.

Con respecto a la cultura material otros autores han dado cuenta, al igual que lo que ocurre en la zona, de la selección de especies según la disponibilidad en las inmediaciones del bosque nativo y de acuerdo con sus propiedades tecnológicas específicas, como dureza, flexibilidad, blandura, entre otros aspectos (Scarpa 2012; Suárez 2014). En este sentido son escasas las referencias etnobotánicas a implementos hípicos como monturas y estribos, siendo un tópico poco abordado en este tipo de estudios por lo que el presente trabajo aportaría datos reveladores. Así, Suárez (2014) da cuenta entre los wichís del bosque xerófito del Chaco Semiárido Salteño del empleo de una sola especie, Pisonia zapallo (Nyctaginaceae) para la confección de monturas y estribos. Por su parte Scarpa (2012) señala para la cultura material hípica de los criollos del oeste formoseño el uso de Cestrum parqui (Solanaceae), Pisonia zapallo (Nyctaginaceae), Ceiba chodatii (Malvaceae) y Caesalpinia paraguariensis (Fabaceae).

\section{Tintóreas}

Se relevaron 24 especies tintóreas pertenecientes a 17 familias botánicas, siendo Fabaceae, la familia con mayor cantidad de representaciones mencionadas (Tabla 1). Esto último coincide con los hallazgos de Paván et al. (2017) y Palacio (2018). En relación con la cantidad de especies, podemos señalar que el nivel es relativamente bajo en comparación con las Yungas donde se hallaron 57 especies (Lambaré et al. 2011), la alta cuenca del Bermejo con 33 especies (Hilgert 2007), la selva misionera con 69 especies (Keller 2010), en la provincia de Santiago del Estero, con 40 especies (Palacio 2018), y el oeste de Córdoba en Guasapampa con 36 especies (Trillo et al. 2007); y superior al norte de Córdoba (Cerro Colorado), donde se hallaron sólo 7 especies (Paván et al. 2017). Esto estaría indicando un intenso proceso de erosión cultural intergeneracional, ya que el área es rica en biodiversidad pero quedan pocos especialistas para ser consultados en la temática. Entre las especies que se reiteran en otros ambientes de Argentina central encontramos el coco, el nogal, el huil, el algarrobo, el granado, el quebracho colorado, la barba de piedra, el amor seco y la pata. Son exclusivos de esta zona el piquillín, el clavillo, la chilca, el arupaco, el piñón, entre otros.
Los primeros tejidos en telares fueron realizados por los indios de las encomiendas, que vestían con telas fabricadas con hilados de algodón. Desde entonces el telar se convirtió en una herramienta muy importante para la industria textil, especialmente en Catamarca (www.atlas.catamarca.gob.ar). En general nuestras observaciones dieron cuenta del empleo de bastidores (para tejer los sobrepelos) y marcos (para hacer alfombras) y para la confección de otras piezas pequeñas como fajas, peleros y caronillas. Menos frecuente es el empleo del telar criollo, el que de acuerdo con Palacio (2018) consta básicamente "de cuatro horcones, clavados en el suelo y que sostienen dos largueros y uno o dos travesaños, el envolvedor (en el que se enrolla la tela terminada) con su sujetador, la "husamanaisana" (a la que se atan los extremos de la urdimbre), el sostén de los hilos de la trama, los lisos, el pisador y el peine, entre otras piezas". En uno solo de los casos constatamos el empleo del gusano de seda obtenida de los Lepidopteros Rothschildia schreiteriana y Rothschildia maurus hilados en huso y teñido, tejido en telar criollo exterior, además del empleo del telar de cintura y bastidor, bordado y tejido con ganchillo, tal como lo señalan Zapata \& Jurado Cazaux (2018 a,b) para Ancasti.

Al igual que lo referido por Palacio (2018), la actividad del telar es sacrificada, pues se realiza a la intemperie o bajo construcciones precarias, empleando para su construcción y la de sus accesorios, partes de vegetales de su entorno que recogen durante el desarrollo de otras actividades o labores domésticas.

Si bien el tipo de material que se tiñe depende de las artesanías a elaborar, las plantas se emplean principalmente para teñir lanas y rara vez hilos de algodón.

La gama de los colores obtenidos a partir de los vegetales es variada: desde los amarillos muy claros, casi color natural, pasando por los verdes, castaños claros, castaños, castaño-rojizos, hasta llegar al marrón oscuro, casi negro (Tabla 1).

La recolección de las especies en Ancasti se realiza alrededor de la vivienda, tal como lo describe Palacio et al. (2007) y Paván et al. (2017) para otras comunidades del Distrito Chaqueño, y en pocos casos, por lo general asociados con adquisiciones recientes de la modernidad, se recurre a productos vegetales adquiridos en los comercios (zanahoria, remolacha, yerba mate). 
El proceso de producción de lanas y tintes, es una actividad manual artesanal donde se prefiere la participación de toda la familia, pero es particularmente la mujer la que lo lleva adelante. En general en el norte argentino, la tarea textil y tintórea también es llevada a cabo por mujeres (Lambaré et al. 2011, Palacio 2007, Suárez \& Arenas 2012, Zapata \& Jurado Cazaux 2018 a,b) y son ellas las que juegan un papel crucial en la adquisición y transmisión de conocimientos (Lambaré et al. 2011, Paván et al. 2017). Es por ello que la desaparición de unos pocos pobladores "clave" (en el sentido de conocer muchas especies y técnicas) podría implicar la pérdida para la comunidad de una importante fuente de información sobre este recurso (Trillo et al. 2007).

La materia prima para teñir consiste en pedazos de madera, corteza de tallo o raíz, resinas, hojas y frutos (Tabla 1). En la preparación se muelen o desmenuzan las partes a utilizar, mientras en otros casos se utilizan los trozos o fragmentos tal como fueron recogidos. El material tintóreo se coloca por lo general en ollas metálicas a las que se agrega el agua y alumbre, luego se somete a hervor para finalmente agregarle la lana. Esta se deja en reposo y luego se enjuaga la lana con agua fría y se coloca a la sombra a secar. Las especies molidas como curtientes en tanto, como el cebil se utilizan depositándolas en un "noque", especie de batea especial, hecho de bloque de piedras.

En relación con el uso de tintes naturales, no es posible afirmar un movimiento lineal hacia el desuso o mayor uso. De hecho, conviven en la región pobladores neorrurales reconocidos por los criollos como "hippies" que han demostrado un creciente interés en el uso de tintes naturales y de hecho lo están comenzando a utilizar, tomando cursos del Instituto Nacional de Tecnología Agropecuaria (INTA) en los que se transmite y enseñan las técnicas. En contraste, una pobladora nativa de la región refería el gran valor de las anilinas sintéticas por la facilidad de su empleo y la intensidad de sus colores, lo que estaría dando cuenta del creciente desuso de plantas tintóreas entre la población criolla, aunque esta hipótesis debería ser corroborada con más encuestas e investigación al respecto.

\section{Conclusiones}

La práctica del teñido natural está todavía vigente en Ancasti y constituye una actividad promisoria si consideramos el creciente interés que va teniendo en las capacitaciones por parte de organizaciones gubernamentales y no gubernamentales que han comenzado a dictarse en la región.
La extracción de material tintóreo podría ser una práctica sustentable que por lo general no impactaría en el entorno ambiental en el que éste se recolecta. La actividad tintórea se encuentra en el conocimiento y manos de pocos pobladores por lo que sería deseable se desarrollen instancias participativas en las que se puedan compartir y hacer circular estos saberes locales. La talabartería, finalmente se encuentra concentrada en menos pobladores aún, que llevan adelante el proceso y producción de materiales de cuero; es deseable que exista un interés renovado en las nuevas generaciones para que se constituyan en aprendices de estas prácticas y oficios para que no se produzca la extinción de la experiencia para lo cual talleres participativos u otras formas de acción serían necesarias.

\section{Declaraciones}

Aprobación ética y consentimiento para participar: se contó con la aprobación de los objetivos del proyecto por parte de la Municipalidad de Ancasti. Además, se estableció un convenio de actividades científicas y de investigación con la Secretaría de Estado del Ambiente y Desarrollo Sustentable, denominado: "Etnobotánica asociada a comunidades rurales: uso y conservación de los recursos naturales en la Sierra de Ancasti" (Expediente No 28950/15). Así mismo se informó a los miembros de la comunidad e individualmente en cada unidad doméstica en la que se solicitó el consentimiento verbal de acuerdo con el código de ética de la Sociedad Internacional de Etnobiología.

Consentimiento para la publicación: No aplica

Disponibilidad de datos y materiales: El material vegetal fue depositado en el Herbario del Museo Botánico (CORD), del Instituto Multidisciplinario de Biología Vegetal (IMBIV), en la Universidad Nacional de Córdoba, Argentina. Se utilizó la serie de numeración N.D. Jiménez-Escobar y G.J. Martínez. Intereses en competencia: Inexistentes Financiamiento: Proyecto Foncyt BID / PICT 1633.

\section{Agradecimientos}

A la población rural de la Sierra de Ancasti que generosamente contribuyó con sus conocimientos. Al Municipio de Ancasti y a la Secretaria de Estado del Ambiente y Desarrollo Sustentable de la Provincia de Catamarca. Al Museo Botánico (IMBIV), al IDACOR (Conicet) y al Conicet. Al Dr. N.D. Jimenez Escobar por atender gentilmente las consultas realizadas.

\section{Literatura citada}

Albuquerque U, Lucena RFP, Alencar NL. 2010. Métodos e técnicas para a coleta de dados etnobiologicos, En: Métodos e técnicas na pesquisa 
etnobiologica e etnoecologica. Editado por UP Albuquerque, RFP Lucena, LVF Cunha. Editora NUPEEA, Recife, Brasil, Pp. 39-63.

Alexiades MN. 1996. Protocol for conducting ethnobotanical research in the Tropics, En Selected guidelines for ethnobotanical research: A field manual. Editado por M.N. Alexiades. New York Botanical Garden. Bronx, New York, Pp. 5-18.

Anderson EN, Persall DM, Hunn ES, Turner NJ (Ed.). 2011. Ethnobiology. John Wiley y Sons Publ., Hoboken-New Jersey, United States.

Arenas P. 2004. Los vegetales en el arte del tatuaje de los indígenas del Gran Chaco. En Los mundos de arriba y los mundos de abajo. Individuo y sociedad en las tierras bajas, en los Andes y más allá. Editado por MS Cipolletti. ABYA YALA, Quito, Ecuador, Pp. 249-274.

Bazán AR. 2006. Los pueblos de Ancasti. Editorial Sarquís, Catamarca, Argentina. 130 pp.

Cantero JJ, Nuñez CO, Bernardello G, Amuchástegui A, Mulko J, Brandolín P, Palchetti MV, Iparraguirre J, VIriginil N, Ariza Espinar L. 2019. Las plantas de interés económico en Argentina. UniRío Editora, Río Cuarto, Argentina. 936 pp.

Hilgert NI. 2007. Plantas silvestres, ámbito doméstico y subsistencia. En Finca San Andrés. Un espacio de conflictos ambientales y sociales. Editado por AD Brown, M García Moritán, BN Ventura, NI Hilgert \& LR Malizia. Ediciones del Subtrópico. Yerba Buena, Tucumán, Argentina, Pp. 187-228.

INDEC. 2010. Instituto Nacional de Estadística y Censos. República de Argentina. En línea:(consultado 01/06/2020; http://www.indec.gov.ar/).

ISE. 2006. International Society of Ethnobiology. Code of Ethics (with 2008 additions). En línea:(consultado 02/06/2020; http://ethnobiology.net/code-of-ethics/).

Jiménez-Escobar ND. 2019. Ciclo de las plantas forrajeras: dinámicas y prácticas de una comunidad ganadera del Chaco Seco, Argentina. Ethnobotany Research \& Applications 18.39:1-22.

Jiménez-Escobar ND. 2019. Etnobotánica asociada al ámbito ganadero: conocimiento, uso y conservación de los recursos vegetales en la Sierra de Ancasti (Catamarca). Tesis de doctorado en Ciencias Biológicas. Universidad Nacional de Córdoba. Argentina. 244 pp.

Jiménez-Escobar ND, Martínez GJ. 2019. Plantas que mantienen al ganado: Conocimiento campesino asociado a especies forrajeras en la Sierra de Ancasti (Catamarca, Argentina). Boletín de la Sociedad Argentina de Botánica 54 (4):617-635.
Joseau MJ, Meehan AR, Bobone A, Hernández R (Eds.), 2018. El uso múltiple del bosque. Producción sostenible de fibras, tejidos y tintes naturales en Argentina. ISPRA, Manuali e linee guida $n$. 171/2018. Roma, Italia.95 pp.

Jurado G, Zapata A. 2002.Textiles realizados con lágrimas de seda nativa de América. En Actas del I Congreso Internacional de Patrimonio Cultural, Córdoba, Pp. 169-183.

Keller H. 2010. Plantas colorantes utlilizadas por los guaraníes de Misiones, Argentina. Bonplandia 19 (1):11-25.

Lambaré DA, Hilgert NI, Ramos RS. 2011. Dyeing Plants and Knowledge Transfer in the Yungas Communities of Northwest Argentina. Economic Botany 65(3):315-328.

Maldonado VP. 2011. Plan Estratégico Territorial de la Provincia de Catamarca: Informe de Avance II: año 2011, 1a ed. Catamarca: Gobierno de Catamarca, Argentina.

Martin G. 2001. Etnobotánica. Manual de Conservación. Serie "Pueblos y Plantas". WWF-UK. UNESCO y Royal BotanicGardens, Kew, Reino Unido. Ed. Nordan Comunidad, Montevideo, Uruguay.

Martínez RD, Fernández EN, Género ER, Rumiano FJ. 2000. El ganado bovino criollo en Argentina. Archivos de Zootecnia 49:353-361.

Marzocca A. 1959. Historia de plantas tintóreas y curtientes. Colección Agropecuaria del INTA. Buenos Aires, Argentina. 234 pp.

Marzocca A. 1993. Index de Plantas colorantes, tintóreas y curtientes. Manual de las especies argentinas. Serie de la Academia Nacional de Agronomía y Veterinaria no. 9. Buenos Aires, Argentina. 334 pp.

Morláns MC. 1995. Regiones Naturales de Catamarca, Provincias Geológicas y Provincias Fitogeográficas. Revista de Ciencia y Técnica. 2(2):1-42.

Musarra A. 2006. Talabartería y zapatería rural. Manual práctico para principiantes. Ed. Maxtor. Valladolid, España.

Palacio M, Carrizo E, Roic L. 2007. Relevamiento de Plantas tintóreas utilizadas en localidades del Dpto. Atamisqui (Santiago del Estero, R. Argentina). Kurtziana 33 (1):1-7.

Palacio M.O. 2018. El uso de plantas tintóreas en Santiago del Estero, Argentina. En: El uso múltiple del bosque. Producción sostenible de fibras, tejidos $y$ tintes naturales en Argentina. Editado por MJ Joseau, AR Meehan, A Bobone, R Hernández. ISPRA, Manuali e linee guida n. 171/2018. Roma, Italia. 
Palmeri CN, Carma IM, Quiroga A. 2008. Las Ecorregiones de Catamarca. Atlas de Catamarca, Universidad Nacional de Catamarca, Facultad de Ciencias Agrarias. http://www.atlas.catamarca.gov.ar En línea (consultado: 17/08/2016).

Paván MF, Furlan V, Renny M, Monterroso I, Argüello L. 2017. Tintes naturales vegetales en el paraje El Desmonte, Reserva Cultural-Natural Cerro Colorado, Córdoba (Argentina). Bonplandia 26(2):103-113.

Scarpa GF. 2012. Las plantas en la vida de los criollos del oeste formoseño. Medicina, Ganadería, Alimentación y Viviendas Tradicionales. Rumbo Sur Ed. Buenos Aires, Argentina, 240 pp.

Stramigioli C. 1991. Teñidos con colorantes naturales. Recuperación de una técnica tradicional. Ediciones Ayllu Búsqueda, Argentina, 156 pp.

Suárez ME. 2014. Etnobotánica wichí del bosque xerófito en el Chaco Semiárido salteño. Autores de Argentina Ed., Buenos Aires, Argentina. 521 pp.

Suárez ME, Arenas P. 2012. Plantas y hongos tintóreos de los wichís del Gran Chaco. Boletín de la Sociedad Argentina de Botánica 47:275-283.

Trillo C, Demaio P, Colantonio S, Galetto L. 2007. Conocimiento actual de plantas tintóreas por los pobladores del Valle de Guasapampa, provincia de Córdoba. Kurtziana Tomo 33 (1):65-71.

Trillo C, Demaio P. 2007. Tintes naturales. Guía para el reconocimiento y uso de plantas tintóreas del Centro de la Argentina. Sezo ed. Argentina. 80 pp.

www.atlas.catamarca.gob.ar/index.php?option=com _content\&view=article\&id=1\&ltemid=262. En línea (Consultado: 14/08/2020).

Zapata A, Jurado Cazaux G. 2018. Polillas y tejidos de seda en bosques nativos de Argentina. Revista de la Facultad de Ciencias Exactas, Físicas y Naturales 5(1):77-82.

Zapata A, Jurado Cazaux G. 2018. Seda silvestre en Argentina: Artesanía e investigación. En: El uso múltiple del bosque. Producción sostenible de fibras, tejidos y tintes naturales en Argentina. Editado por MJ Joseau, AR Meehan, A Bobone, R Hernández. ISPRA, Manuali e linee guida n. 171/2018. Roma, Italia. 